\title{
REVIEW CONCERNING THE CONDITIONING AND RECONDITIONING OF DRILLING SHIPS
}

\author{
Violeta-Elena PAVELIU, Gabriel-Marius DUMITRU \\ Politehnica University of Bucharest, Romania \\ e-mail: elena.stefanescu16@gmail.com
}

\begin{abstract}
The drill bit consists of: heavy rods, drill rods, drive rod, reducers and fittings. After a certain number of operating hours for a subassembly, some parts, more difficult to request, have an advanced state of wear, which makes it impossible to continue functioning of the respective assembly. In the tubular bases, the rods brought from the yard are identified, sorted and pre-directed. If they are clogged, they are cleaned with very long drills. For a good cleaning, the rods are left for a time in a room containing a solution of detergents and caustic soda. Then the rods are washed on the outside and inside, with water jets and rotary plastic fiber brushes. Afterwards, they are dried with hot air. After correcting their rectilinearity, with hydraulic presses, the actual control begins. Measure the length, outer diameter, wall thickness and, using the methods below, detect any operating defects.
\end{abstract}

KEYWORDS: Drilling gasket, reconditioning, Reconditioning by welding

\section{Introduction}

The drill bit consists of: heavy rods, drill rods, drive rod, reducers and fittings. The heavy rods are at the bottom of the gaskets, the drilling poles at the middle of them have the longest length, and the drive rod at the top of the gasket.

Drilling rods are tubes of steel, aluminum, or light alloy (titanium). In order to increase the capacity of resistance to joints, their ends are thickened (reinforced). Cutting the thread in this case does not weaken the strength of the rod [1].

\section{Reconditioning of used parts}

After a certain number of operating hours for a subassembly, some parts, more difficult to request, have an advanced state of wear, which makes it impossible to continue functioning of the respective assembly.

There are also accidental malfunctions due to which the machines may be out of service. Some of these reasons could be: overloads, hits, incorrect mountings, defects of material, etc.

It can be observed that, after disassembly, the WEIGHT DEGREE is established (the analysis of the parts, measurements, comparisons, checks from where the nature and size of the wear results). This degree of wear is the factor that directly influences the cost of reconditioning, it helps us to assess correctly whether or not to resort to reconditioning [2].

\section{Reconditioning of parts through machining on machine tools}

This process is carried out in an organized framework, after the elaboration of an economic technological process, which is expressed by the technological processing documentation, where all the necessary technological operations are prescribed. The technological process is carried out following the technological sheet and the operations plan [3].

- TECHNICAL DATA SHEET - a standardized form in which we can read all the data of the technological process.

- OPERATION PLAN - establishes the sequence of operations and work phases (each operation on a tab).

The wear can be compensated by the execution and introduction of bushings, with tightening, or fixed by other procedures such as threading, small fixing welds, threaded pins, etc.

Analysing a little the technology of reconditioning the teeth of this wheel (not the whole 
wheel, with radial beats, wear on the hub, etc.) for success we need to do:

- Washing, thickening, measuring.

- Complete removal of the defective part, until the remaining material has no defect. joints.

- Weaving the edges of the rupture, cracks,

- Loading of the used surface by successive deposition of metal layers (electrode chosen according to the chemical composition of the piece material).
- Final mechanical processing, which ensures the dimensions required in the drawing and the quality of the surfaces.

When the parts have high wear (for example a rack) or have been reconditioned several times (for example by welding and then processing), they can be remedied by removing the worn area mechanically (by cutting or heat) and applying instead of an additional part. For example, some racks, from the design, are made of several pieces, being very long. They are worn unevenly, especially on certain portions, so the total replacement is not justified [4].

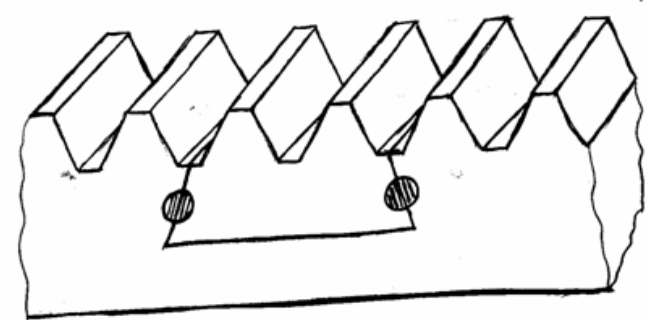

Fig. 1. Reconditioning of a rack by removing an area and replacing it [4]

\section{Reconditioning by welding}

Welding is one of the most used reconditioning procedures. In addition to the fact that we have many welding procedures available, a wide range of repairs can be provided:

- compensation of wear by metal deposits;

- reconditioning of cracks, cracks or breaks;

- combining broken pieces;

- joining component parts to a welded device or construction.

A repair using a specific welding process is indicated to meet the following conditions:

- the resistance of the piece in the welding area to approach that of the base material;

- the addition metal should be as close as chemical composition and mechanical properties to that of the part;

- the layer or the deposited cord will then allow processing by cutting to correct the shape and size [5]. BENEFITS:

- allows the reconditioning of parts that have a lot of workmanship;

- material economy;

- low cost;

- repairs are made that cannot be done by other procedures;

- the equipment used for welding is simple, cheap, with great possibility of diversification;

- welding is easily suitable for machining and automation.
DISADVANTAGES:

- the structure of the joint usually differs from that of the base material;

- remaining tensions that can cause deformations or even breakages in operation [6].

\section{Reconditioning of parts by soldering}

The method of reconditioning the pieces by soldering is applied in case of breaks, cracks, cracks or it is necessary to replace a part of the used part.

The process is used for joints of parts that are not subjected to high demands. There is a mutual diffusion on the surfaces of the base metal (the soldering parts) and the alloy for soldering, there is an exchange of atoms between the two materials resulting in a bonding alloy in the form of an intermediate layer [7].

It is necessary to prepare the parts before the soldering operation, in order for the jointing place to be well cleaned of fats, dirt, oxides, etc. The main operations that are performed before gluing are:

Mechanical cleaning - (wire brush, grinding, blasting, polishing, scraping, etc.).

Degreasing - (gasoline, petroleum, sodium hydroxide, sodium carbonate, sodium silicate).

Pickling - (sulfuric, hydrochloric or phosphoric acid solutions).

Depending on the melting temperature of the soldering alloys, it is possible to apply soft soldering (with alloys having a melting temperature below 500 ${ }^{\circ} \mathrm{C}$ ), and hard soldering (with alloys having melting 
temperatures above $500{ }^{\circ} \mathrm{C}$, such as AmSiLp and AmSnLp alloys) [8].

\section{Control and conditioning of drilling rigs}

In the tubular bases, the rods brought from the yard are identified, sorted and pre-directed. If they are clogged, they are cleaned with very long drills. For good cleaning, the rods are left for a time in a chamber containing a solution of detergents and caustic soda. Then the rods are washed on the outside and inside, with water jets and rotary brushes made of plastic fibers. Afterwards, they are dried with hot air [9].

After correcting the rectilinearity with hydraulic presses, the actual control begins. Measure the length, outer diameter, wall thickness and, using the methods below, detect any operating defects. The area where the pens are fixed is very important. Where the electromagnetic inspection signals an invisible defect through a voltage peak (the defect can be inside the wall), the area is thoroughly cleaned and magnetic particles are used: the method has a much higher resolution. Defects on the inner surface (corrosion attacks or erosion traces) can be located by an optical technique, with reflection mirrors [10].

Rods that are identified as having cracks are removed. Some superficial defects can be identified by simple grinding.

The controlled rods are classified in the classes corresponding to the size of the outer diameter, the thickness of the wall and the relative value of the various defects.

Sometimes rods are tested for traction and internal pressure [11].

\section{Conclusions}

Heavy rods, reductions and drive rods are generally controlled after one month of work. If one or three cracks are detected at the base of the spirals, the inspection interval is appropriate. If the number of cracks is large, checks should be performed more often.

Modern procedures also take into account the growth rate of fatigue cracks. The calculation shows that the working time of an element from the moment of detection of cracks until its rupture is relatively short.

According to the Romanian norms, the control periods are fixed in rotation hours and vary within the quite wide limits, depending on the importance of the controlled element, the wear class, the control place (probe, base), the complexity of the control and the total duration of use ( the intervals decrease in time): 300-2500 h. Of course, a strict record of working time is required for each element in the gasket.

\section{References}

[1]. ***, https://ro.wikipedia.org/wiki/Garnitur\%C4\%83_de_foraj. [2]. Ionescu M., Nicolescu S., Probe drilling technology, Ed. IPG, Ploiesti, 2003.

[3]. Radoi M., Huzum N. et al., Reconditioning of parts, Ed. Tehnica, Bucuresti, 1986.

[4]. Neculai Macovei, Drilling of wells 2, Drilling equipment, Ed. IPG, Ploiesti, 1996.

[5]. ***, https://www.sciencedirect.com/topics/engineering.

[6]. ***, https://www.scribd.com.

[7]. ***, https://www.scribd.com/doc/316309691/Garnitura-deForaj.

[8]. ***, http://www.ondrill.ro/blog/general/prăjini-de-foraj/.

[9]. Lazăr Avram, Curs Forajul dirijat.

[10]. Neculai Macovei, Drilling of wells, ed. UPG Ploiesti, 2014.

[11]. Iordache G., Auxiliary works in drilling-extraction, Editura Tehnică, Bucuresti, 1979.

[12]. Macovei N., Drilling of wells - 1-Drilling fluids and wells, Editura Editura Tehnica, Bucuresti, 1974. 